\title{
The WT1-like transcription factor
}

2 Klumpfuss maintains lineage commitment

3

4

5

6

7

8

9

10

11

12 Jerome Korzelius ${ }^{1 \S}$, Tal Ronnen-Oron ${ }^{2}$, Maik Baldauf $^{1}$, Elke Meier ${ }^{1}$, Pedro

13 Sousa-Victor ${ }^{2}$, and Heinrich Jasper ${ }^{1,2,3 \S}$

14

15 1. Leibniz Institute on Aging - Fritz Lipmann Institute (FLI), Jena, Germany

16 2. Buck Institute for Research on Aging, 8001 Redwood Boulevard, Novato,

17 CA 94945-1400, USA

18 3. Immunology Discovery, Genentech, Inc., 1 DNA Way, South San

Francisco, CA 94080, USA

20

$21 \S($ Co-)Corresponding author

22 Jerome.Korzelius@leibniz-fli.de jasper.heinrich@gene.com 


\section{Abstract}

Stem cell (SC) lineages in barrier epithelia exhibit a high degree of plasticity.

Mechanisms that govern the precise specification of SC daughter cells during regenerative episodes are therefore critical to maintain homeostasis. One such common mechanism is the transient activation of the Notch $(\mathrm{N})$ signaling pathway. $\mathrm{N}$ controls the choice between absorptive and entero-endocrine cell fates in both the mammalian small intestine and the Drosophila midgut, yet how precisely $\mathrm{N}$ signaling promotes lineage restriction in progenitor cells remains unclear. Here, we describe a role for the WT1-like transcription factor Klumpfuss (Klu) in restricting the fate of Drosophila enteroblasts (EBs) downstream of $\mathrm{N}$ activation. Klu is transiently induced in Notch-positive EBs and its transient activity restricts cell fate towards the enterocyte (EC) lineage.

Transcriptomics and DamID profiling show that Klu suppresses enteroendocrine (EE) cell fates by repressing E(Spl)m8-HLH and Phyllopod, both negative regulators of the proneural gene Scute, which is essential for EE differentiation. At the same time, Klu suppresses cell cycle genes, committing EBs to differentiation. Klu-mediated repression of its own

transcription further sets up a negative feedback loop that ensures temporal restriction of Klu-mediated gene regulation, and is essential for subsequent differentiation of ECs. Our findings define a transient cell state in which EC lineage restriction is cemented, and establish a hierarchy of transcriptional programs critical in executing a differentiation program downstream of initial induction events governed by $\mathrm{N}$ signaling. 
71 Notch signaling plays a central role in both ISC proliferation and lineage

72 differentiation. ISCs produce the Notch-ligand Delta and activate Notch in the

\section{Introduction}

In many tissues, somatic SCs respond to tissue injury by increasing their proliferative potential and producing new differentiating cell progeny. To maintain homeostasis during such periods of regeneration, cell specification and differentiation need to be precisely coordinated within a dynamic environment. Studies in the mammalian intestine have demonstrated a surprising plasticity in such specification events, showing that even differentiated cells can revert into a stem cell state in conditions in which tissue homeostasis is perturbed ${ }^{1,2}$. These findings highlight the critical role of gene regulatory networks in establishing and maintaining differentiated and committed cell states in homeostatic conditions.

The Drosophila midgut is an excellent model to study lineage differentiation of adult stem cells both in homeostasis as well as during regeneration and aging. The Drosophila midgut is maintained by intestinal stem cell (ISCs), which can generate differentiated enterocytes $(E C)$ or entero-endocrine $(E E)$ cells ${ }^{3,4}$. Upon injury or infection, ISC proliferation is dramatically increased in response to mitogenic signals from damaged enterocytes ${ }^{5-7}$. Mis-regulation of cell specification and differentiation in this lineage can lead to significant dysfunction, as evidenced in aging intestines, where disruption of normal $\mathrm{N}$ signaling due to elevated Jun-N-terminal Kinase (JNK) signaling leads to an accumulation of mis-differentiated cells that contribute to epithelial dysplasia and barrier dysfunction ${ }^{8,9}$. 
73 EB daughter cell. Levels of Delta vary markedly between ISCs in the

74 homeostatic intestine. These differences have been proposed to underlie the

75 decision between EC and EE differentiation in a specific ISC lineage ${ }^{10}$. It has

76 been proposed that higher $\mathrm{N}$ activity is associated with differentiation into the

77 EC fate, while lower $\mathrm{N}$ activity promotes $\mathrm{EE}$ differentiation ${ }^{10,11}$. Loss of $\mathrm{N}$ in

78 ISC lineages leads to the formation of tumors that consist of highly Delta-

79 expressing ISCs and of Prospero (Pros)-expressing EEs ${ }^{10,12,13}$. These tumors

80 are likely a consequence of impaired EB differentiation, resulting in an

81 increased frequency of symmetric divisions, as well as of differentiation of a

82 subset of EBs into EE, suggesting that EE differentiation is a default fate in

83 the lineage when $\mathrm{N}$ signaling activity is reduced.

84 Interestingly, recent work has shown that lineage specification in ISC

85 daughter cells is likely more complex than a simple model in which EB fates

86 are determined stochastically or by 'lateral-inhibition' - like N-mediated

87 mechanisms. It has been shown that pre-determined ISCs exist that express

88 the EE marker Prospero and generate daughter cells that differentiate into

89 EEs ${ }^{14,15}$.

90 The exact cell state in which the decision between EE and EC fates is

91 cemented, however, remains unclear. A transient specification step has been

92 identified in EE differentiation, in which cells transiently express Scute, a

93 transcription factor that negatively regulates $\mathrm{N}$ responsive genes such as

$94 \mathrm{E}(\mathrm{Spl}) \mathrm{m8}$, as well as its own expression ${ }^{16}$. Furthermore, EBs have been

95 shown to remain in a transient state for a prolonged period of time before

96 differentiating into an EC fate ${ }^{17}$. Mechanisms that regulate and maintain this

97 transient state remain unclear. 
99 Here we describe a role for the Wilms' Tumour 1 (WT1) - like transcription

100 factor Klumpfuss (Klu) in lineage commitment during EC differentiation in the

101 adult fly intestine. In transcriptome studies of FACS sorted ISCs and EBs, we

102 found Klu to be expressed in an Esg-dependent manner specifically in EBs.

103 Klu is critical for leg and bristle development, as well as for lineage

104 differentiation of Type II neuroblast stem cells ${ }^{18,19}$. Klu is related to the

105 mammalian tumor suppressor gene Wilms' Tumour 1 (WT1), and its

106 overexpression in neuroblast stem cells leads to tumorous overgrowths that

107 can grow autonomously when transplanted in the abdomen of flies ${ }^{20,21}$. In the

108 intestine, we find that loss of Klu leads to aberrant entero-endocrine

109 differentiation of EB cells, whereas ectopic activation of Klu results in a failure

110 to differentiate. Transcriptomics and DNA-binding studies reveal that Klu

111 controls EE differentiation by repressing genes involved in Notch signaling, as

112 well as by indirectly controlling the levels of the Achaete-Scute complex

113 members asense and scute. Klu further represses its own expression,

114 defining a transient state of EBs in which specification into ECs is cemented

115 by precise temporal regulation of $\mathrm{N}$ signaling. We propose that the transient

116 expression of Klu 'locks in' the EC fate in EBs, preventing ectopic proneural

117 gene activation and thus ensuring lineage commitment into the $\mathrm{EC}$ fate. 


\section{Results}

120 The transcription factor Klu is expressed in the enteroblast precursor

121 cells

122 We identified Klumpfuss (Klu) transcripts to be specifically enriched in

123 transcriptomes isolated from sorted progenitor cells ${ }^{22}$ and to be significantly

124 downregulated upon loss of the stem and progenitor specific transcription

125 factor Escargot (Esg) ${ }^{23}$. To confirm and better characterize klu expression in

126 the Drosophila posterior midgut, we used a klu-Gal4, UAS-GFP reporter line

127 that reflects Klu expression in the wing and eye discs of wandering 3rd instar

128 larvae $^{18,24}$. In the midgut, GFP expression was seen in the larger cells of the

129 stem-progenitor nests (ISC+EB). These cells resemble EBs based on both

130 nuclear and cellular size (Figure 1A arrowheads). To confirm their identity, we

131 combined the Klu reporter line with the Notch activity reporter Su(H)GBE-lacZ,

132 which is exclusively activated in EBs, but not in ISCs ${ }^{10}$. In addition, we used

133 Delta-lacZ (DI-lacZ) as a marker for ISCs. The expression of klu-Gal4, UAS-

134 GFP overlapped almost exclusively with the EB-specific Su(H)GBE-lacZ

135 reporter. In contrast, ISC-specific Delta-lacZ staining was mostly found in

136 small, diploid cells neighboring the GFP-positive cells (Figure $1 \mathrm{~B}, \mathrm{C}$,

137 quantification in D, E).

138 Lineage tracing experiments have previously shown that Notch-positive EB

139 precursor cells exclusively give rise to enterocytes, whereas Delta-positive

140 ISCs can give rise to clones with both ECs and EEs ${ }^{14,15}$. To trace the fate of

141 Klu-expressing cells, we crossed the klu-Gal4 enhancer-trap line to a Actin

142 promoter-driven FlipOut lineage tracing cassette (UAS-GFP, tub-

143 Gal80ts; UAS-Flp, Act>STOP>Gal4). Upon temperature shift, the UAS-FIp- 
144 inducible $A c t>S T O P>$ Gal4 will be activated, marking all cells that express Klu

145 as well as all differentiated progeny from these cells. As expected, DI-Gal4

146 expressing ISCs give rise to both ECs (visible as large GFP-positive cells with

147 a large, polyploid nucleus) as well as EEs, marked by expression of the

148 transcription factor Prospero (Pros) (Figure 1F, arrows). In contrast, Notch-

149 positive EBs (Su(H)GBE-Gal4) only gave rise to ECs, but not EEs (Figure 1G,

150 arrowheads). Similar to Notch-positive EBs, klu-Gal4-traced cells gave rise

151 exclusively to ECs, but not EEs (Figure $1 \mathrm{H}$ ). We conclude that Klu is

152 specifically expressed in the EC-generating EBs in the Drosophila midgut.

153

154 Klu loss of function leads to excess EE differentiation

155 To determine the role of Klu in the specification and/or differentiation of cells

156 in the ISC lineage, we first inhibited Klu function using RNAi. We used the

157 TARGET-system to express UAS-driven RNAi constructs in specific lineages

158 in a temperature-dependent manner ${ }^{25}$. We used the esg-Gal4 ${ }^{\text {ts }}$ driver to

159 express Klu RNAi in ISCs and EBs and the Su(H)GBE-Gal4 ${ }^{\text {ts }}$ driver that drives

160 expression in EBs only. In both conditions, knockdown of Klu lead to an

161 increase of EEs in the posterior midgut (Figure 2A-D, quantification in E). To

162 confirm the EE differentiation phenotype, we used Mosaic analysis with a

163 repressible cell marker (MARCM) ${ }^{26}$ to generate marked lineages

164 homozygous for a null allele of Klu, $k l u^{R 5118}$ and traced the fate of $k l u^{R 51}$

165 mutant cells. Quantification showed that loss of Klu leads to more EE

166 cells/clone (Figure 2F-I). Interestingly, the GFP-negative tissue also contained

167 more EEs in $k l u^{R 51}$ MARCM animals than in the control animals (FRT2A,

168 Figure $2 \mathrm{~F}$, compare with $2 \mathrm{G}$ ). This is likely due to the fact that in this 
169 genotype, the GFP-negative tissue is heterozygous for the $k / u^{R 51}$ null allele. In

170 addition, we generated stem cell clones expressing $k / u^{R N A i}$ using the escargot

171 promoter-driven FlipOut system (esg-F/O). Enterocyte differentiation still

172 occurred in esg-F/O $>k / u^{R N A i}$ clones based on nuclear size of cells within the

173 clones and staining for the enterocyte marker Pdm1 ${ }^{23,27}$ (Figure S1).

174 In summary, these results indicate that loss of Klu function alters the EE-to-

175 EC ratio, but does not impair EC differentiation.

176

177 Ectopic Klu expression inhibits stem cell differentiation and impairs

178 Delta-Notch signaling

179 We hypothesized that constitutive Klu expression would impair EE

180 differentiation. To test this, we used the esg-F/O system to express full-length

181 Klu in ISC-derived clones. Wild-type esg-F/O clones take up most of the

182 posterior midgut 2 weeks after induction, containing a mixture of ECs and EEs

183 (Figure 3A). In contrast, clones expressing full-length Klu did not exhibit any

184 hallmarks of differentiation into either EEs or ECs, but contained only a few

185 small, round cells (Figure 3B). Klu is thought to act mainly as a repressor of

186 transcription, based on studies in neuroblast stem cells and in sensory organ

187 precursor (SOP) cells in the developing wing ${ }^{20,24,28}$. To ask whether this

188 repressor function of Klu would elicit the phenotypes observed, we expressed

189 the zinc-finger DNA-binding domain of Klu fused to either a VP16 activation

190 domain (Klu-VP16) or fused to the repressor domain from Engrailed (Klu-

191 ERD) ${ }^{28}$. Whereas differentiation still occurred in clones expressing the

192 activating Klu-VP16, differentiation was significantly impaired in clones

193 expressing the repressing Klu-ERD, confirming that transcriptional repression 
194 of genes regulated by Klu is sufficient to impair differentiation (Figure 3C-D,

195 quantification in E).

196 Together with the temporally restricted endogenous expression pattern of Klu,

197 these data indicate that Klu is acting in early EBs to restrict EE differentiation,

198 but that it has to be downregulated and that its sustained expression keeps

199 cells in an undifferentiated state. To specify this state, we combined UAS-Klu

200 with the ISC-marker DI-lacZ and the EB-marker Su(H)GBE-lacZ. Interestingly,

201 esg-F/O clones expressing UAS-klu did not stain positive for either DI-lacZ or

$202 \mathrm{Su}(H) G B E-l a c Z$. This suggests that Klu expression promotes an exit from the

$203\left(\mathrm{DI}^{+}\right)$stem cell state, but also interferes with transcriptional programs induced

204 by Delta-Notch signaling in EBs. To further investigate the interaction between

205 Notch signaling and Klu activity in ISC differentiation, we performed epistasis

206 experiments: the formation of large tumors consisting of proliferating ISCs and

207 Pros-positive EEs in $\mathrm{N}$ loss of function conditions could be prevented by

208 expression of Klu (UAS-klu expression in $N^{R N A i}$ esg-F/O clones), which

209 resulted in a complete block of both cell proliferation and EE differentiation

210 (Figure S2). This suggests that Klu acts downstream of Notch in EC

211 differentiation, but additionally acts as a potent inhibitor of cell proliferation.

212 We further combined expression of Klu (UAS-Klu) with expression of the

213 oncogenic Ras $^{V 12}$ variant (UAS-Ras ${ }^{V 12}$ ) in esg-F/O clones. Whereas esg-F/O>

$214 \operatorname{Ras}^{\mathrm{V} 12}$ clones occupy the entire posterior midgut 2 days after induction and

215 contribute to a significant loss of viability of the animal, co-expression of UAS-

216 klu markedly reduced clonal size at this timepoint and rescued viability (Figure

217 S3). 
218 Altogether, our results indicate that the $\mathrm{N}$-mediated transient expression of

219 Klu in EBs is critical to restrict lineage commitment to the EC fate and inhibit

220 proliferation, but that normal differentiation can only proceed once Klu is

221 downregulated. To test this hypothesis, and to better understand the

222 mechanism by which transient Klu expression controls EB cell fate, we

223 decided to explore the progenitor cell-specific transcriptional program

224 downstream of Klu.

225

226 Transcriptome profiling supports a role for Klu in regulating Notch

227 signaling and EE fate repression

228 To gain a comprehensive overview of the genes controlled by Klu in the

229 intestine, we performed RNA-Sequencing on biological triplicate populations

230 of FACS sorted Esg-positive progenitor cells in which we expressed either a

$231 k / u^{R N A i}$ construct or UAS-klu (see Figure 4A, Supplementary Figure S5 and

232 Methods for details). To perform these studies, we used an approach

233 previously described in ${ }^{29}$. We first confirmed that the transcriptome of sorted

234 cells indeed reflects the excess EE differentiation phenotype seen in $k / u^{R N A i}$

235 animals by performing qRT-PCR for prospero (pros) and scute (sc). The EE

236 marker pros is upregulated 5-fold upon $k l u^{R N A i}$ (Figure 4B). The proneural

237 transcription factor Scute $(s c)$ is necessary and sufficient for EE generation in

238 the Drosophila midgut ${ }^{14,30,31}$ and many upstream factors impinge on the

239 expression of Sc to regulate EE differentiation ${ }^{32}$. mRNA levels of $s c$ increase

$240 \sim 2.5$-fold upon $k / u^{R N A i}$ animals and UAS-klu expression completely abolishes

$241 s c$ mRNA expression in stem-progenitor cells (Figure 4B). 
242 In addition, we checked the mRNA levels for Klu to verify knockdown and

243 overexpression efficiency. As expected, we see a $70 \%$ reduction in $\mathrm{mRNA}$

244 levels upon $k l u^{R N A i}$. Surprisingly, we found that the expression of $k l u$ mRNA in

245 UAS-klu-expressing progenitor cells was almost completely abolished

246 compared to control (Figure 4C). This was contrary to our expectation of

247 detecting increased expression upon Gal4/UAS-mediated overexpression, but

248 was explained by the fact that the UAS-klu construct does not carry the

249 endogenous klu 3'UTR, which our primers targeted. Accordingly, primers that

250 target the coding region of $k l u(k / u C D S)$ readily detect a $\sim 12$-fold upregulation

251 of klu transcript. Hence, while transgenic Klu was induced as expected, the

252 endogenous Klu gene was repressed, indicating that Klu may repress its own

253 expression. The notion of a negative autoregulatory loop was confirmed in our

254 RNA-seq data, as we saw increased reads in the CDS of the gene, and no

255 reads in the 3' UTR (Figure S5).

256 Comparing the transcriptomes of wild-type progenitors with the experimental

257 samples, we found 410 genes significantly upregulated in $k / u^{R N A i}$ and 809

258 genes significantly dowregulated in UAS-klu-expressing esg ${ }^{+}$cells $($Padj <

$2590.05, \log _{2} \mathrm{FC}>0.5$ or $\left.<-0.5\right)$. Given the role of Klu as a repressor of

260 transcription, we focused our RNA-Seq data analysis on genes that would be

261 upregulated in the absence of Klu, but downregulated upon UAS-klu

262 expression in Esg-positive stem-progenitor cells (Figure 4D). In this category

263 of 81 genes, many genes involved in the regulation of Notch signaling (the

$264 \mathrm{HES} / E(S p /)$ - complex genes $m 6, m 7, m 8$, and the HES-like transcription factor

265 Deadpan), as well as several previously described regulators of EE

266 differentiation (encoding the proneural proteins Asense (ase), Scute (sc), and 
267 the adaptor protein Phyllopod, phyl)) could be identified (Figure 4E).

268 Additional $E(S p l)$ genes $(E(S p l)-m \delta$ and $E(S p l)-m y)$ were significantly

269 upregulated in $k / u^{R N A i}$ samples, but did not significantly change in UAS-klu

270 samples (Figure 4E). E(Spl)-genes are a group of genes activated by Notch

271 that mediate its downstream transcriptional response ${ }^{33}$. Phyl, in turn, acts to

272 destabilize Tramtrack (ttk), a strong repressor of the achaete-scute complex

273 genes scute and asense, and loss of which leads to a dramatic increase in EE

274 numbers ${ }^{3234}$. Reciprocally, loss of phyl stabilizes Ttk and results in a

275 complete loss of EE cells in the midgut ${ }^{35}$. The induction of phyl in klu loss of

276 function conditions thus explains the increase in EEs.

277 We also found that the expression of the transcription factor Charlatan (chn) is

278 significantly downregulated by UAS-klu expression. Chn is a transcription

279 factor that positively regulates the proneural genes Achaete and Scute and

280 loss of Chn in the midgut leads to proliferation and differentiation defects in

281 the stem-progenitor compartment ${ }^{36-38}$. Hence, Klu represses the expression

282 of several genes that have reported roles in EE differentiation.

283 Our transcriptome data also revealed transcriptome changes downstream of

284 Klu that may explain the Klu-induced exit from the stem cell state: stem cell

285 maintenance is dependent on the Class I bHLH-family member Daughterless

286 (Da)/E47-like, since loss of da results in loss of ISC fate and differentiation

287 into enterocytes ${ }^{31}$. The gene miranda (mira) is a Da/proneural target gene

288 that is also highly expressed in ISCs ${ }^{31,38}$. Proneural factors such as Ase and

289 Sc require Da to dimerize and regulate transcription ${ }^{39}$. We found that loss of

290 Klu results in a slight but significant upregulation of mira mRNA, whereas Klu

291 overexpression results in a 2.3-fold downregulation (Figure 4E). To confirm 
292 this, we used the mira-Promoter-GFP (mira-GFP) line and combined this with

$293 k / u^{R N A i}$ and UAS-klu. Confocal microscopy and FACS-sorting of cells

294 expressing either $k / u^{R N A i}$ and UAS-klu confirmed that UAS-klu expression

295 could reduce mira-GFP levels whereas a slight induction is seen in $k / u^{R N A i}$

296 cells (Figure 4F-I).

297 Taken together, we conclude that Klu has three main functions in EBs: 1) To

298 turn off the Notch transcriptional response by repressing $\mathrm{E}(\mathrm{Spl})$-gene

299 expression, 2) to promote the exit from the stem cell state by repressing

300 genes like mira, and 3) To repress aberrant EE differentiation in the EB by

301 repressing proneural gene activation. By responding to $\mathrm{N}$ activity and

302 repressing its own expression, Klu further serves as a 'timer' for N-mediated

303 transcriptional responses in EBs.

304

305 Klu acts upstream of the transcription factor Scute in EE differentiation

306 Our genetic and transcriptional profiling experiments suggest that Klu likely

307 acts downstream of Notch, but upstream of the proneural genes Ase and Sc

308 in repressing EE differentiation (Figure 3, Figure 4, Figure S2). Scute plays a

309 critical role in a transcriptional loop that regulates both ISC proliferation and

310 the initiation of EE differentiation ${ }^{16}$. Our data indicate that Klu can act to

311 inhibit both proliferation and EE differentiation by affecting factors that

312 genetically act upstream of Scute (Figure 3 and Figure 4). Therefore, we

313 performed epistasis experiments with Klu and Sc to confirm this hypothesis.

314 We generated esg-F/O clones that express $k / u^{R N A i}$ in the presence or absence

315 of $s c^{R N A i}$. Clones expressing $k l u^{R N A i}$ contained more EE cells compared to

316 control clones (Figure 5A-B), whereas clones expressing $s c^{R N A i}$ are almost 
317 completely devoid of EE cells (Figure $5 C$ ). The combination of $k / u^{R N A i}$ and

$318 s c^{R N A i}$ also resulted in clones with little or no EE differentiation, as quantified

319 by the number of Pros-positive cells (Figure 5D, quantification in 5E). This

320 suggests that the appearance of extra EE cells in $k l u^{R N A i}$-expressing clones

321 depends on Scute. To confirm that Scute would act downstream of Klu in

322 determining EE fate, we combined overexpression of Scute and Klu. Clonal

323 expression of Scute using the esg-F/O system resulted in clones consisting

324 almost entirely of Pros-positive EE cells whereas clones expressing UAS-klu

325 are completely devoid of EE cells (Figure 5F, Figure S6A-D). Co-expression

326 of Klu and Scute leads to a marked reduction in clone size (Figure S6F) but

327 EE differentiation was observed in a large fraction of the clones, although the

328 percentage of differentiated cells is reduced compared to UAS-Sc alone

329 (Figure 5F). We conclude that Scute can still induce EE differentiation, even in

330 Klu gain-of-function conditions.

331 We observed an increase in the number of Pros-pH3 double-positive cells in

332 UAS-Sc compared to control, likely representing the EE-progenitor cells (EEp)

333 undergoing a final round of division ${ }^{16}$. Strikingly, this percentage increased in

334 esg-F/O > UAS-klu+UAS-sc clones (Figure S6E). Since the clonal size in this

335 genotype is no larger than in esg-F/O>UAS-klu single overexpression clones

336 (Figure S6F), indicating that these cells might be arrested in mitosis. This

337 suggests that although Klu expression cannot completely repress UAS-Sc-

338 induced EE differentiation, the effect of Klu on cell cycle progression interferes

339 with the proliferation-inducing capacity of Scute. 
342 Klu directly represses several genes that regulate EE fate, Notch

343 signaling and the cell cycle

344 To identify genes that are directly regulated by Klu, we performed targeted

345 DamID of Klu in esg-positive stem-progenitor cells ${ }^{40}$. We used the DamID-

346 seq pipeline ${ }^{41}$, see Methods) to identify 1667 genes that had 1 or more Klu

347 binding peak(s) within $2 \mathrm{~KB}$ of their gene body in all 3 replicates. Using 2

348 published position weight matrices for Klu-binding ${ }^{42}$, we could establish that

349692 of the 1667 genes $(41.5 \%)$ had 1 or more Klu-binding motif(s) present in

350 their binding peaks. We considered these peaks as high-confidence Klu-

351 bound sites. Our transcriptomics data on Klu indicated that Klu controls many

352 genes involved in Notch signaling, EE differentiation and cell cycle regulation.

353 We identified a cluster of binding sites at the centrosomal end of the $E(S p l)$ -

354 locus around the $E(S p l)-m \delta$ and $E(S p l)-m y$ genes (Figure 6E). Since our

355 RNA-Seq data showed that many of the $E(S p l)$-genes change expression in

356 both $k / u^{R N A i}$ and UAS-klu conditions (Figure 4E), this suggests that Klu could

357 possibly regulate the expression of multiple members of the $E(S p /)$-complex

358 through this binding peak at the centrosomal end of the $E(S p l)$-locus.

359 Furthermore, we identified a Klu-Dam binding peak at the klu locus,

360 supporting our hypothesis that Klu acts in an autoregulatory loop by

361 negatively regulating its own expression (Figure 6A). Previous work has

362 shown that Scute and the $E(S p l)$-complex member $E(S p l) m 8-H L H$ act in a

363 regulatory loop to generate an EE precursor directly from the ISC ${ }^{16}$. Since

364 our results indicate that Scute is upregulated upon loss of Klu and acts

365 epistatically to Klu in EE formation, we first looked for Klu binding in and

366 around the scute locus. We did not find significant binding of Klu-Dam around 
367 any of the genes in the Achaete/Scute complex. However, we did identify a

368 DamID peak around the sina and sinah loci (Figure 6B). Together with the

369 adaptor protein Phyllopod, the Sina and Sinah E3-ubiquitin ligases are able to

370 degrade the transcriptional repressor Tramtrack (ttk), which represses EE fate

$371 \quad 32,35$. sina transcript levels are upregulated 2.2-fold upon klu RNAi and phyl

372 levels are upregulated 8-fold as well as downregulated 15-fold upon UAS-klu

373 expression (Figure 4E). Hence, we propose that Klu represses EE-fate

374 determination in EBs upstream of Scute by stabilizing Tramtrack, since Klu

375 directly represses the members of the E3-ligase complex Sina, Sinah and

376 (indirectly) Phyl that can normally target Ttk for destruction.

378 In addition to genes involved in Notch signaling and EE-specification, we find

379 evidence for direct repression of critical cell cycle regulators by Klu. We find

380 Klu binding peaks at both the Cyclin $\mathrm{B}(\mathrm{CycB})$ and Cyclin $\mathrm{E}(\mathrm{CycE})$ loci

381 (Figure 6C and D), two Cyclins that are essential for G1-S and G2-M

382 progression respectively. CycE is also upregulated upon klu RNAi expression.

383 Notch activation is essential for the mitotic-to-endocycle switch in follicle cells

384 of the Drosophila ovary, and polyploidization is a critical step in the normal

385 process of EB-to-EC differentiation ${ }^{43,44}$. We propose that Klu plays a role in

386 remodeling the cell cycle in response to Notch activation by directly

387 repressing 2 critical cell cycle regulators. Furthermore, this explains how Klu

388 acts as a potent suppressor of cell proliferation, even in combination of the

389 strongly oncogenic RasV12 overexpression (Figure 3 and Figure S3). 
391 Altogether, our data suggest a model (Figure 6G) in which Klu acts as a Notch

392 effector in the EB that acts to restrict the duration of the Notch transcriptional

393 response (through negative regulation of the $\mathrm{E}(\mathrm{Spl})$-complex members and

394 Klu itself). Second, Klu prevents activation of the Scute-E(Spl)m8

395 transcriptional circuit that triggers EE differentiation. Finally, we find evidence

396 that Klu can bind and repress critical cell cycle regulators such as Cyclin B

397 and Cyclin E, likely promoting the switch from a mitotic to an endoreplicating

398 cell cycle in differentiating ECs.

400 


\section{Discussion}

402 Our work identified a mechanism by which lineage decisions are cemented

403 through the coordinated repression of alternative fates and of cell proliferation

404 in somatic stem cell daughter cells. N-induced expression of Klu in EBs is

405 necessary to repress EE fates in EBs, but also to temporally restrict $\mathrm{N}$ target

406 gene expression. Hence, its own expression has to be self-regulated to allow

407 differentiation to ECs to proceed. We find that Klu represses several genes

408 that are critical for EE differentiation; most notably genes that influence the

409 accumulation of the transcription factor Scute. Transient expression of Scute

410 is necessary and sufficient for EE differentiation and the transient nature of

411 Scute activation is accomplished by a double-negative feedback loop between

412 Scute and $\mathrm{E}(\mathrm{Spl}) \mathrm{m} 8{ }^{16}$. The expression of Klu results in the repression of both

413 transcription factors in EBs, inactivating the transcriptional circuit that governs

414 EE differentiation (Figure 6F). Previous work on Klu has shown that Klu is

415 directly regulated by $\mathrm{Su}(\mathrm{H})$ and acts as a Notch effector in hemocyte

416 differentiation ${ }^{45}$. In the ISC lineage, we find that overexpression of Klu results

417 in the loss of Notch signaling activity in progenitor cell clones, and that Klu is

418 able to repress several Notch effector genes (such as the HES/E(Spl) family

419 and HES/E(Spl)-like genes such as Deadpan). We thus propose that Klu acts

420 in a negative feedback loop downstream of Notch signaling to ensure that

421 Notch effector gene activity is transient in EBs, mirroring the transient nature

422 of EE specification by Scute and E(Spl)m8.

423 Loss of WT1 in the mouse kidney results in glomerulosclerosis and is

424 accompanied by ectopic expression of HES/E(Spl) family genes ${ }^{46}$ and in

425 zebrafish kidney podocytes Notch expression induces Wt1 transcription, while 
426 the Notch intracellular domain (NICD) and WT1 synergistically promote

427 transcription at the promoter of the HES/E(Spl) family gene Hey ${ }^{47}$. This

428 suggests that the negative feedback between Notch and its effector Klu/WT1

429 might be conserved between species, even though conservation at the

430 sequence level between these transcription factors is low.

431 Our data also support a role for Klu for regulating cell cycle progression.

432 Overexpression of Klu results in a strong block in cell proliferation in $N^{R N A i}$ or

433 oncogenic Ras ${ }^{V 12}$-induced tumors and our DamID binding studies suggest

434 that Klu can directly regulate Cyclins B and E. This is in stark contrast to its

435 role in the neuroblast stem cell lineage, where overexpression of Klu leads to

436 a strong overproliferation of immature neural progenitor cells and the resulting

437 formation of brain tumors that can be transplanted to distant regions of the

438 body and continue to grow ${ }^{20,21}$. However, this likely reflects the different role

439 for Notch in the NB lineage, where continuous activation of Notch also leads

440 to INP overproliferation and tumor formation. Thus, the role of Klu in

441 promoting either lineage differentiation or stem-progenitor cell proliferation

442 seems to be context-dependent. This is reminiscent of the context-specific

443 rules for Notch as either a tumor suppressor or an oncogene in mammalian

444 adult stem cell lineages. For instance, activating Notch1 mutations are the

445 main driver of T-cell acute lymphoblastic leukemia ${ }^{48}$, whereas Notch-

446 inactivating mutations are found in many epithelial-derived solid tumor types

447 such as head-and-neck or skin carcinomas (see ${ }^{49}$ and references therein).

448 Similarly, Wt1 was initially identified as a tumor-suppressor gene mutated in

449 the rare pediatric kidney cancer Wilms' Tumor ${ }^{50}$. Recently, WT1 was also

450 identified as a strong inhibitor of proliferation in a genome-wide gain-of- 
451 function screen for tumor suppressors and oncogenes ${ }^{51}$. However,

452 expression of WT1 was found to be elevated in many solid tumors and in

453 acute myeloid leukemia ${ }^{52,53}$. Thus, similar to Notch, the role of WT1 in

454 tumorigenesis seems to be highly context-dependent. Intriguingly, in both

455 nephric and hematopoietic lineages WT1 is often transiently expressed in

456 committed progenitor cells, similar to the expression of Klu in the EB

457 progenitors, raising the possibility that to fully understand the role of WT1-like

458 proteins in tumorigenesis, cell lineage relationships, as well as cell

459 proliferation and differentiation events in tumors need to be taken into

460 account.

461 Critically, our work highlights the role for transient transcriptional 'rewiring'

462 events during cell specification in somatic stem cell lineages. Such events

463 seem to be required to ensure lineage commitment downstream of initial

464 symmetry breaking signals like Notch, and ensure progression of cell

465 differentiation into a defined lineage. As such, it can be expected that similar

466 transcriptional feedback loops need to be reversed for cells to undergo de-

467 differentiation into stem cells in mammalian regenerating tissues. We propose

468 that understanding their makeup and regulation will significantly advance

469 efforts to control tissue repair and regeneration in mammals, including

470 humans. 


\section{Methods}

\section{Fly strains and husbandry}

473 The following strains were obtained from the Bloomington Stock Center:

474 BL28731 (klu RNAi on 3rd) BL60469 (klu RNAi on 2nd), BL56535 (UAS-

$\left.475 k u^{[H t o]}\right), \mathrm{BL} 11651$ (D/ ${ }^{05151}$-lacZ) BL26206 (sc RNAi), BL51672 (UAS-sc),

476 BL1997 (w[*]; $P\{w[+m W . h s]=F R T(w[h s])\} 2 A)$, BL4540 (w[*]; $P\{w[+m C]=U A S-$

477 FLP.D\}JD2). BL65433 (y[1] $w\left[^{\star}\right] ; M\{w[+m C]=h s . \min (F R T . S T O P 1) d a m\} Z H-$

478 51C) BL1672 (w[1118]; sna[Sco]/CyO, P\{ry[+t7.2]=en1\}wg[en11]).

479 VDRC: v27228 (N RNAi). Other stocks: klu-Gal4 UAS-GFP, FRT2A

480 kluR51/Tm6B, hs-Flp, Tub-Gal4, UAS-GFP/Fm7;FRT2A,

481 TubGal80ts/Tm2,Ubx (T. Klein, Düsseldorf) UAS-kluFL, UAS-ERD-kluZF,

482 UAS-VP16-kluZF (C.Y. Lee, U. Michigan) esg-F/O (w; esg-Gal4, tub-Gal80ts,

483 UAS-GFP; UAS-flp, Act>CD2>Gal4(UAS-GFP)/TM6B), esg ${ }^{t s}(y, w ; e s g-G a l 4$,

484 UAS-GFP/CyO;tub-Gal80ts/Tm3), Su(H) ${ }^{t s}$ (w;Su(H)GBE-Gal4,UAS-CD8-

485 GFP/CyO;tub-Gal80 ${ }^{\text {ts }} / T M 3$ ), ISC-specific esg ${ }^{\text {ts }}$ (Wang et al., 2014) w;esg-

486 GAL4,UAS-2XEYFP/CyO;Su(H)GBE-GAL80,tub-Gal80ts/TM3,Sb, w;esg-

487 gal4, tub-Gal80ts, UAS-GFP/CyO,wg-lacZ;P\{w[+mC]=UAS-FLP.D\}JD2/Tm6B.

488 Stocks generated in this study: w;Klu-Dam(ZH-51C) M4M1/CyO,

$489 P\{r y[+t 7.2]=e n 1\} w g[e n 11]$

490

491 Immunostaining and microscopy

492 Midgut immunostaining was performed as described in ${ }^{54}$. Antibodies used

493 include: Chicken anti-GFP (1:1000, ThermoFisher A10262), mouse anti-

494 Prospero (MR1A, 1:50, DSHB), mouse anti- $\beta$-galactosidase (40-1a, 1:200,

495 DSHB), rabbit anti- $\beta$-galactosidase (1:200, ThermoFisher A11132) mouse 
496 anti-Armadillo (N2 7A1, 1:20, DSHB), rabbit anti-phosphorylated Histone H3-

497 Ser10 (pH3S10, 1:500, sc8656-R, Santa Cruz Biotechnology). Images were

498 taken from the R5 and R4 regions of the posterior midgut on a Zeiss Apotome

499 microscope or Zeiss LSM710 confocal at either 20X or 40X magnification.

500 Images were captured as Z-stacks with 8-10 slices of 0.22-1.0 $\mu \mathrm{m}$ thickness.

501 Images were converted to maximum-intensity projections in Fiji (https://fiji.sc)

502 and quantifications were performed using the CellCounter FiJi plugin. Scale

503 bar $=50 \mu \mathrm{m}$ in all images, except in Figure 1A: scale bar $=25 \mu \mathrm{m}$. Graphing,

504 statistical analysis and survival curves were produced in GraphPad Prism.

505

506 Cloning and transgene generation

507 We used the Inducible DamID system from the Van Steensel lab to generate

508 klu-Dam ${ }^{40}$. To this end, we amplified the Klu Full-length cDNA (derived from

509 BDGP Gold clone FI01015) using Ascl and Notl-containing primers and

510 cloned the fragment into the vector $p$-attB-min.hsp70P-FRT-STOP\#1-FRT-

511 DamMyc[open] (Addgene plasmid \#71809). Transgenic lines were generated

512 by Genetivision Inc. using the phiC31 integrase-mediated site-specific

513 transgenesis system ${ }^{55}$. The finished construct was injected into Bloomington

514 stock BL24482 ( $\mathrm{ZH}-51 \mathrm{C}$ attP-site on $2 \mathrm{nd})$ and the resulting transgenic lines

515 were tested by genotyping PCR. Both control (Dam-only, BL65433) and klu-

516 Dam transgenic lines were crossed to BL1672 (w[1118]; sna[Sco]/CyO,

$517 P\{r y[+t 7.2]=e n 1\} w g[e n 11])$ before use. 
520 Control Dam-only (BL65433) and klu-Dam male flies were crossed to w;esg-

521 gal4, tub-Gal80ts, UAS-GFP/CyO,wg-lacZ;P $\{w[+m C]=U A S-F L P . D\} J D 2 / T m 6 B$

522 virgins. Crosses were maintained at $18^{\circ} \mathrm{C}$ and progeny was shifted to $29^{\circ} \mathrm{C}$ for

52324 hours to induce the FIp-mediated recombination of the STOP-Cassette.

$52430-50$ midguts of Dam-only and klu-Dam were dissected in 1X PBS in 3

525 different batches and used for isolation of total genomic DNA. Isolation of

526 methylated GATC-sequences and subsequent amplification was done

527 according to the protocol published by Marshall et al. ${ }^{56}$ until Step 34, from

528 which we continued NGS library preparation using the Illumina TruSeq nano

529 DNA kit LT. After library quality control, samples were sequenced as 50 bp

530 single-end on an Illumina HiSeq2500.

532 Midgut FACS, RNA-isolation and Sequencing

533 Midgut dissociation and FACS was performed as described in ${ }^{29}$. UAS-

534 expression of UAS-klu or $k / u^{R N A i}$ was induced for 2 days, followed by 16 hours

535 of Ecc15 infection to stimulate midgut turnover. We dissected 100

536 midguts/genotype in triplicate and for each sample 20,000-40,000 cells were

537 sorted into RNAse-free 1X PBS with 5 mM EDTA. RNA was isolated using the

538 Arcturus PicoPure ${ }^{\mathrm{TM}}$ RNA Isolation Kit. Subsequently, the entire amount of

539 isolated RNA was used as input for RNA-amplification using the Arcturus ${ }^{\mathrm{TM}}$

540 RiboAmp ${ }^{\text {TM }}$ HS PLUS Kit. 200 ng of amplified aRNA was used as input for

541 RNA-Seq library preparation using the TruSeq Stranded mRNA Library Prep

542 Kit (Illumina) and samples were subsequently sequenced as 50 bp single-end

543 on an Illumina HiSeq2500. 
545 Quantitative real-time PCR

546 Quantitative real-time PCR (qRT-PCR) was performed using aRNA from

547 FACS-sorted $\mathrm{esg}^{+}$cell populations (see above) as template. qRT-PCR was

548 performed using the TaqMan FAM-MGB system in a $10 \mu$ reaction on a

549 BioRad CFX384 C1000 Touch Cycler using the following probes: klu

550 (dm02361358 s1), pros (dm02135674 g1), sc (dm01841751 s1) and Act5C

551 (dm02361909 s1) was used for normalization. The klu CDS primer assay was

552 ordered as a Custom TaqMan Assay. Reactions were performed in triplicate

553 on 3 independent biological replicates. Relative expression was quantified

554 using the ${ }^{{ }^{\Delta}} \mathrm{Ct}$ method. Data were calculated using Microsoft Excel and

555 plotted as relative fold-changes +/- SEM in Graphpad Prism.

556

557 RNA-Seq and DamID data analysis

558 The 15-21 million quality-passed reads per sample were mapped to the $D$.

559 melanogaster reference genome (BDGP6) with TopHat2 (version 2.1.0) ${ }^{57}$. Of

560 each sample, approximately $80 \%$ of the reads was mapped to the genome.

561 From this, 90\% could be assigned to genes using FeatureCounts resulting in

562 11-15 million analysis-ready reads per sample ${ }^{58}$.

563 The table of raw counts per gene/sample was analyzed with the $\mathrm{R}$ package

564 DESeq2 (version 1.16.1) for differential expression ${ }^{59}$. Both sample groups of

565 interest (UAS \& RNAi) were pair-wise contrasted with the control sample

566 group (control). For each gene of each comparison, the p-value was

567 calculated using the Wald significance test. Resulting p-values were adjusted

568 for multiple testing with Benjamini \& Hochberg correction. Genes with an

569 adjusted $p$-value $<0.05$ are considered differentially expressed (DEGs). 
570 For DamID, we used the damid_seq pipeline ${ }^{41}$ to genrate binding profiles for

571 Klu-Dam. Triplicate samples for $\operatorname{Klu}$-Dam (34.9, 33.5 and 34.1 millions reads)

572 and Dam-only control (34.7, 34.5, and 35.6 million reads) were aligned to the

573 Drosophila genome (UCSC dm6). Overall aligning rate was between $86 \%$ and

$57491 \%$ across all samples. First, 'gat.track.maker.pl' script was used to build a

575 GATC fragment file. Then the main utility 'damidseq_pipeline' was used to

576 align the reads to the genome using bowtie2, bin and count reads, normalize

577 counts, and compute log2 ratio between corresponding DamID and control

578 Dam-only samples. The pipeline identified 1,707, 1,663, 1,681 peaks with

579 FDR $<0.01$ per each replicate. To test for reproducibly we first used Marshall

580 OJ's damid_pipeline to identify peaks with weaker confidence (FDR< 0.1$)$ and

581 the 'idr' python package (https://github.com/nboley/idr) to identified 1,169

582 peaks with IDR<0.05 between replicate1 and replicate2. We used an in-house

583 developed script to annotate peaks in proximity to genes. 1,667 genes found

584 to be in proximity to at least one reproducible peak. To find Klu binding motifs

585 in our reproducible peak set, we scanned for 2 different Klu PWM (described

586 in $^{42}$ ) around reproducible peaks using the FIMO tool ${ }^{60}$. Reads were

587 visualized using IGV as overlayed triplicate Klu-Dam ( $\log _{2}$ FC over Dam-only)

588 tracks. 


\section{Acknowledgements}

591 The authors would like to thank Thomas Klein, C.Y. Lee, Sarah Bray, Claude

592 Desplan, Benoit Biteau, Cai Yu and Bruce Edgar for providing fly stocks and

593 antibodies, the Bloomington Drosophila Stock Center, The VDRC (Vienna)

594 and the Developmental Studies Hybridoma Bank (DSHB) for fly stocks and

595 antibodies, and Maria Locke, Marco Groth, Philipp Koch and Karol Szafranski

596 from the Flow Cytometry, Sequencing and Bioinformatics Core Facilities at

597 the FLI-Leibniz Institute on Aging for expert technical assistance. This work

598 was supported by DFG research grant number KO5594/1-1 to J.K.

599

\section{Author contributions}

601 J.K and H.J conceived the project and designed experiments. J.K., M.B. and

602 E.M. performed experiments and collected data. T.R-O. performed data

603 analysis on the RNA-Seq and DamID samples. P.S-V. provided preliminary

604 data for the study. J.K. and H.J. wrote the manuscript.

605

606 Competing interests

607 The authors declare no competing financial interests.

608

609

610 
612

613

614

615

616

617

618

619

620

621

622

623

624

625

626

627

628

629

630

631

632

633

634

635

636

637

638

639

640

641

642

643

644

645

646

647

648

649

650

651

652

653

654

655

656

657

658

659

\section{Bibliography}

1. Tetteh, P. W. et al. Replacement of Lost Lgr5-Positive Stem Cells through Plasticity of Their Enterocyte-Lineage Daughters. Cell Stem Cell 18, 203213 (2016).

2. Yan, K. S. et al. Intestinal Enteroendocrine Lineage Cells Possess

Homeostatic and Injury-Inducible Stem Cell Activity. Cell Stem Cell 21, 78-90.e6 (2017).

3. Micchelli, C. A. \& Perrimon, N. Evidence that stem cells reside in the adult Drosophila midgut epithelium. Nature 439, 475-479 (2006).

4. Ohlstein, B. \& Spradling, A. The adult Drosophila posterior midgut is maintained by pluripotent stem cells. Nature 439, 470-474 (2006).

5. Buchon, N., Broderick, N. A., Poidevin, M., Pradervand, S. \& Lemaitre, B. Drosophila intestinal response to bacterial infection: activation of host defense and stem cell proliferation. Cell Host Microbe 5, 200-211 (2009).

6. Jiang, $\mathrm{H}$. et al. Cytokine/Jak/Stat signaling mediates regeneration and homeostasis in the Drosophila midgut. Cell 137, 1343-1355 (2009).

7. Biteau, B., Hochmuth, C. E. \& Jasper, H. JNK activity in somatic stem cells causes loss of tissue homeostasis in the aging Drosophila gut. Cell Stem Cell 3, 442-455 (2008).

8. Li, H. \& Jasper, H. Gastrointestinal stem cells in health and disease: from flies to humans. Dis Model Mech 9, 487-499 (2016).

9. Choi, N.-H., Kim, J.-G., Yang, D.-J., Kim, Y.-S. \& Yoo, M.-A. Age-related changes in Drosophila midgut are associated with PVF2, a PDGF/VEGFlike growth factor. Aging Cell 7, 318-334 (2008).

10. Ohlstein, B. \& Spradling, A. Multipotent Drosophila intestinal stem cells specify daughter cell fates by differential notch signaling. Science 315 , 988-992 (2007).

11. Perdigoto, C. N., Schweisguth, F. \& Bardin, A. J. Distinct levels of Notch activity for commitment and terminal differentiation of stem cells in the adult fly intestine. Development 138, 4585-4595 (2011).

12. Patel, P. H., Dutta, D. \& Edgar, B. A. Niche appropriation by Drosophila intestinal stem cell tumours. Nat Cell Biol 17, 1182-1192 (2015).

13. Siudeja, K. et al. Frequent Somatic Mutation in Adult Intestinal Stem Cells Drives Neoplasia and Genetic Mosaicism during Aging. Cell Stem Cell 17, 663-674 (2015). 
660

661

662

663

664

665

666

667

668

669

670

671

672

673

674

675

676

677

678

679

680

681

682

683

684

685

686

687

688

689

690

691

692

693

694

695

696

697

698

699

700

701

702

703

704

705

706

707

708

709

14. Zeng, X. \& Hou, S. X. Enteroendocrine cells are generated from stem cells through a distinct progenitor in the adult Drosophila posterior midgut. Development 142, 644-653 (2015).

15. Biteau, B. \& Jasper, H. Slit/Robo signaling regulates cell fate decisions in the intestinal stem cell lineage of Drosophila. Cell Rep 7, 1867-1875 (2014).

16. Chen, J. et al. Transient Scute activation via a self-stimulatory loop directs enteroendocrine cell pair specification from self-renewing intestinal stem cells. Nat Cell Biol 20, 152-161 (2018).

17. Antonello, Z. A., Reiff, T., Ballesta-Illan, E. \& Dominguez, M. Robust intestinal homeostasis relies on cellular plasticity in enteroblasts mediated by miR-8-Escargot switch. EMBO J 34, 2025-2041 (2015).

18. Klein, T. \& Campos-Ortega, J. A. klumpfuss, a Drosophila gene encoding a member of the EGR family of transcription factors, is involved in bristle and leg development. Development 124, 3123-3134 (1997).

19. Yang, X., Bahri, S., Klein, T. \& Chia, W. Klumpfuss, a putative Drosophila zinc finger transcription factor, acts to differentiate between the identities of two secondary precursor cells within one neuroblast lineage. Genes Dev 11, 1396-1408 (1997).

20. Xiao, Q., Komori, H. \& Lee, C.-Y. klumpfuss distinguishes stem cells from progenitor cells during asymmetric neuroblast division. Development 139, 2670-2680 (2012).

21. Berger, C. et al. FACS purification and transcriptome analysis of drosophila neural stem cells reveals a role for Klumpfuss in self-renewal. Cell Rep 2, 407-418 (2012).

22. Sousa-Victor, P. et al. Piwi is required to limit exhaustion of aging somatic stem cells. Cell Rep 20, 2527-2537 (2017).

23. Korzelius, J. et al. Escargot maintains stemness and suppresses differentiation in Drosophila intestinal stem cells. EMBO J 33, 2967-2982 (2014).

24. Kaspar, M., Schneider, M., Chia, W. \& Klein, T. Klumpfuss is involved in the determination of sensory organ precursors in Drosophila. Dev Biol 324, 177-191 (2008).

25. McGuire, S. E., Mao, Z. \& Davis, R. L. Spatiotemporal gene expression targeting with the TARGET and gene-switch systems in Drosophila. Sci STKE 2004, pl6 (2004).

26. Lee, T. \& Luo, L. Mosaic analysis with a repressible cell marker (MARCM) for Drosophila neural development. Trends Neurosci 24, 251-254 (2001). 
27. Lee, W.-C., Beebe, K., Sudmeier, L. \& Micchelli, C. A. Adenomatous polyposis coli regulates Drosophila intestinal stem cell proliferation. Development 136, 2255-2264 (2009).

28. Janssens, D. H. et al. An Hdac1/Rpd3-Poised Circuit Balances Continual Self-Renewal and Rapid Restriction of Developmental Potential during Asymmetric Stem Cell Division. Dev Cell 40, 367-380.e7 (2017).

29. Dutta, D., Xiang, J. \& Edgar, B. A. RNA expression profiling from FACSisolated cells of the Drosophila intestine. Curr Protoc Stem Cell Biol 27, Unit 2F.2. (2013).

30. Amcheslavsky, A. et al. Enteroendocrine cells support intestinal stem-cellmediated homeostasis in Drosophila. Cell Rep 9, 32-39 (2014).

31. Bardin, A. J., Perdigoto, C. N., Southall, T. D., Brand, A. H. \& Schweisguth, F. Transcriptional control of stem cell maintenance in the Drosophila intestine. Development 137, 705-714 (2010).

32. Wang, C., Guo, X., Dou, K., Chen, H. \& Xi, R. Ttk69 acts as a master repressor of enteroendocrine cell specification in Drosophila intestinal stem cell lineages. Development 142, 3321-3331 (2015).

33. Delidakis, C., Monastirioti, M. \& Magadi, S. S. E(spl): genetic, developmental, and evolutionary aspects of a group of invertebrate Hes proteins with close ties to Notch signaling. Curr Top Dev Biol 110, 217262 (2014).

34. Pi, H., Huang, S.-K., Tang, C.-Y., Sun, Y. H. \& Chien, C.-T. phyllopod is a target gene of proneural proteins in Drosophila external sensory organ development. Proc Natl Acad Sci U S A 101, 8378-8383 (2004).

35. Yin, C. \& Xi, R. A Phyllopod-Mediated Feedback Loop Promotes Intestinal Stem Cell Enteroendocrine Commitment in Drosophila. Stem Cell Reports 10, 43-57 (2018).

36. Escudero, L. M., Caminero, E., Schulze, K. L., Bellen, H. J. \& Modolell, J. Charlatan, a Zn-finger transcription factor, establishes a novel level of regulation of the proneural achaete/scute genes of Drosophila.

Development 132, 1211-1222 (2005).

37. Amcheslavsky, A. et al. Gene expression profiling identifies the zinc-finger protein Charlatan as a regulator of intestinal stem cells in Drosophila.

Development 141, 2621-2632 (2014).

38. Reeves, N. \& Posakony, J. W. Genetic programs activated by proneural proteins in the developing Drosophila PNS. Dev Cell 8, 413-425 (2005).

39. Cabrera, C. V. \& Alonso, M. C. Transcriptional activation by heterodimers 
of the achaete-scute and daughterless gene products of Drosophila. EMBO J 10, 2965-2973 (1991).

40. Pindyurin, A. V., Pagie, L., Kozhevnikova, E. N., van Arensbergen, J. \& van Steensel, B. Inducible DamID systems for genomic mapping of chromatin proteins in Drosophila. Nucleic Acids Res 44, 5646-5657 (2016).

41. Marshall, O. J. \& Brand, A. H. damidseq_pipeline: an automated pipeline for processing DamID sequencing datasets. Bioinformatics 31, 33713373 (2015).

42. Nitta, K. R. et al. Conservation of transcription factor binding specificities across 600 million years of bilateria evolution. elife 4, (2015).

43. Shcherbata, H. R., Althauser, C., Findley, S. D. \& Ruohola-Baker, H. The mitotic-to-endocycle switch in Drosophila follicle cells is executed by Notch-dependent regulation of G1/S, G2/M and M/G1 cell-cycle transitions. Development 131, 3169-3181 (2004).

44. Xiang, J. et al. EGFR-dependent TOR-independent endocycles support Drosophila gut epithelial regeneration. Nat Commun 8, 15125 (2017).

45. Terriente-Felix, A. et al. Notch cooperates with Lozenge/Runx to lock haemocytes into a differentiation programme. Development 140, 926-937 (2013).

46. Asfahani, R. I. et al. Activation of podocyte Notch mediates early Wt1 glomerulopathy. Kidney Int 93, 903-920 (2018).

47. O'Brien, L. L. et al. Wt1a, Foxc1a, and the Notch mediator Rbpj physically interact and regulate the formation of podocytes in zebrafish. Dev Biol 358, 318-330 (2011).

48. Weng, A. P. et al. Activating mutations of NOTCH1 in human T cell acute lymphoblastic leukemia. Science 306, 269-271 (2004).

49. Ntziachristos, P., Lim, J. S., Sage, J. \& Aifantis, I. From fly wings to targeted cancer therapies: a centennial for notch signaling. Cancer Cell 25, 318-334 (2014).

50. Call, K. M. et al. Isolation and characterization of a zinc finger polypeptide gene at the human chromosome 11 Wilms' tumor locus. Cell 60, 509-520 (1990).

51. Sack, L. M. et al. Profound tissue specificity in proliferation control underlies cancer drivers and aneuploidy patterns. Cell (2018). doi:10.1016/j.cell.2018.02.037

52. Qi, X. et al. Wilms' tumor 1 (WT1) expression and prognosis in solid 
cancer patients: a systematic review and meta-analysis. Sci Rep 5, 8924 (2015).

53. Huff, V. Wilms' tumours: about tumour suppressor genes, an oncogene and a chameleon gene. Nat Rev Cancer 11, 111-121 (2011).

54. Resnik-Docampo, M. et al. Tricellular junctions regulate intestinal stem cell behaviour to maintain homeostasis. Nat Cell Biol 19, 52-59 (2017).

55. Bischof, J., Maeda, R. K., Hediger, M., Karch, F. \& Basler, K. An optimized transgenesis system for Drosophila using germ-line-specific phiC31 integrases. Proc Natl Acad Sci U S A 104, 3312-3317 (2007).

56. Marshall, O. J., Southall, T. D., Cheetham, S. W. \& Brand, A. H. Cell-typespecific profiling of protein-DNA interactions without cell isolation using targeted DamID with next-generation sequencing. Nat Protoc 11, 15861598 (2016).

57. Kim, D. et al. TopHat2: accurate alignment of transcriptomes in the presence of insertions, deletions and gene fusions. Genome Biol 14, R36 (2013).

58. Liao, Y., Smyth, G. K. \& Shi, W. featureCounts: an efficient general purpose program for assigning sequence reads to genomic features. Bioinformatics 30, 923-930 (2014).

59. Love, M. I., Huber, W. \& Anders, S. Moderated estimation of fold change and dispersion for RNA-seq data with DESeq2. Genome Biol 15, 550 (2014).

60. Grant, C. E., Bailey, T. L. \& Noble, W. S. FIMO: scanning for occurrences of a given motif. Bioinformatics 27, 1017-1018 (2011). 
845 Figure legends

846 Figure 1. The transcription factor Klu is specifically expressed in

847 enteroblast cells. A-A' The klu-Gal4, UAS-GFP reporter line shows

848 expression in the midgut epithelium. ISCs (arrows) and EBs (arrowheads) are

849 visualized by esg-lacZ. Cells are outlined with Armadillo/ $\beta$-catenin. B-C. The

850 klu-Gal4, UAS-GFP was combined with Su(H)-GBE-lacZ (enteroblast (EB)

851 marker) or DI-lacZ (intestinal stem cell (ISC) marker). Expression of Klu

852 largely overlaps with the EB marker Su(H)-GBE-lacZ (B), and Klu-positive

853 cells are found adjacent to the Delta-positive ISC (C). D-E. Quantification of

854 marker gene overlap of the genotypes displayed in (B-C) $n=7$ guts (DI-lacZ,

$855 n=1370$ cells counted) and $n=10$ guts (Su(H)-GBE-lacZ, $n=572$ cells

856 counted). F-H. Lineage-tracing of cells in the intestine using different cell-

857 specific drivers. F-F'. The DI-Gal4-positive ISCs give rise to both differentiated

858 cell types of the intestinal lineage (enterocytes (EC) and entero-endocrine

859 (EE) cells). EEs are marked by antibody staining for the transcription factor

860 Prospero (Pros, in red). Arrows indicate GFP-Pros double-positive EEs in the

861 clonal area, whereas arrowheads indicate EEs outside the clonal area. G-G'.

$862 \mathrm{Su}(\mathrm{H})-\mathrm{GBE}-$ positive EB cells exclusively give rise to ECs, but not to EEs

863 (arrowheads). H-H'. Similar to Su(H)-GBE-positive EBs, Klu-positive cells also

864 give rise exclusively to ECs, but not EEs.

865

866 Figure 2. Loss of Klu leads to excess EE differentiation. A-E. RNAi-

867 mediated knockdown of Klu results in an excess of Pros-positive EE-cells.

868 Expression of $k l u^{R N A i}$ using either the EB-specific $S u(H) G B E-G a l 4^{t s}$ driver

869 (Pros in red, compare A with B) or the ISC+EB driver esg-Gal4 ${ }^{\text {ts }}$ (Pros in 
870 green, compare $\mathrm{C}$ with $\mathrm{D})$ results in more $\mathrm{EE}$ cells in the posterior midgut. $\mathbf{E}$.

871 EE cell quantification of the posterior midgut for the genotypes in A-D. Error

872 bars represent mean +/- S.D. Significance was calculated using Student's t-

873 test with Welch's correction. Number of midguts $n=15$ (control $\left.w^{1118}\right)$ and $n=$

$87418\left(k / u^{R N A i}\right)$ for experiments with the $S u(H) G B E-G a l 4^{t s}$ driver and $n=12$

875 (control $\left.w^{1118}\right)$ and $n=16\left(k / u^{R N A i}\right)$ for esg-Gal4 ${ }^{t s}$ F-I. Clonal analysis of the

$876 k / u^{R 51}$ null mutant allele using the MARCM technique. $k / u^{R 51}$ mutant clones

877 have more EE cells compared to FRT2A control clones (compare F and G).

878 H-I. Quantification of the number of Pros-positive EEs/clone $(H)$ and the total

879 number of Pros-positive EEs/ROI for the genotypes in F-G. $n=15$ guts

880 (FRT2A control) and $n=17$ guts $\left(k / u^{R 51}\right)$. Error bars represent mean +/- S.D.

881 Significance was calculated using Student's t-test with Welch's correction.

882

883 Figure 3. Klu overactivation results in a loss of intestinal stem cell

884 differentiation and Delta-Notch signaling. A-D. Clonal expression of

885 different Klu isoforms using the esg-FlipOut (esg-F/O) system to generate ISC

886 clones. A. Control esg-FO clones grow to occupy most of the posterior midgut

8872 weeks after clonal induction. B-D. Clones expressing either full-length Klu

888 (UAS-kluFL, B) or the Klu zinc-finger DNA-binding domain fused to the

889 Engrailed repressor domain (UAS-ERD-kluZF, D) resulted in a block of

890 differentiation. This was not observed when expressing the Klu zinc-finger

891 DNA-binding domain fused to the VP16 transcriptional activator domain (UAS-

892 VP16-kluZF, C). E. Quantification of genotypes in A-D. Error bars represent

893 mean +/- S.D. Significance was calculated using Student's t-test with Welch's

894 correction. $n=5$ for each genotype. F-I. Klu expression in ISC clones leads to 
895 a loss of Notch signaling activity in ISC-EB pairs. F. Control clones always

896 contain 1 or more ISCs that are positive for the Notch ligand Delta (DI-lacZ,

897 red). G. DI-lacZ staining is absent from clones expressing UAS-kluFL. H.

898 Control clones have EB cells that are marked by presence of the Notch

899 activity reporter $S u(H)-G B E-l a c Z$ (red). I. Notch transcriptional activity is

900 absent from UAS-kluFL-expressing esg-F/O clones.

901

902 Figure 4. Transcriptome profiling indicates that Klu acts to repress

903 Notch signaling output and genes critical to EE differentiation. A.

904 Overview of the experiment: $\operatorname{ssg}^{t s} \mathrm{GFP}^{+}$cells either expressing $k / u^{R N A i}$ or

905 UAS-kluFL were sorted in triplicate and their transcriptome was compared to

906 control esg ${ }^{\text {ts }}$ GFP cells (see Methods for more details). B. qRT-PCR analysis

907 of sorted cells for Klu and the critical EE fate regulators Scute (sc) and

908 Prospero (pros). C. qRT-PCR analysis of klu mRNA expression with a primer

909 pair that targets the endogenous 3' UTR coding sequence (klu UTR) and a

910 primer pair that targets the coding region only (klu CDS). D. Overlap of

911 significantly upregulated genes in $k l u^{R N A i}$ and significantly downregulated

912 genes in UAS-kluFL. E. Volcano plots comparing expression of a selection of

913 genes from the overlap of 81 genes shown in (D). Genes upregulated in the

$914 k / u^{R N A i}$ VS control set (left) are downregulated in the UAS-klu VS control set

915 (right). F-I. Klu represses Da-dependent miraP-GFP expression in ISC and

916 EB. Control miraP-GFP expression (F) is high in ISCs and EBs and slightly

917 increased in $k / u^{R N A i}$ midguts (G). UAS-kluFL expression in reduced levels of

918 miraP-GFP $(\mathrm{H})$. I. GFP-intensity of of miraP-GFP-positive cells for the

919 genotypes in (F-H) by FACS. $n=50$ midguts per genotype. 
921 Figure 5. Scute acts downstream of Klu in the induction of entero-

922 endocrine differentiation. A-D. Expression of $k / u^{R N A i}$ lead to increased EE

923 differentiation in clones 14 days after clonal induction, marked by increased

924 numbers of Pros ${ }^{+}$-cells (red) (A-B, quantification in E-F). sc ${ }^{R N A i}$ clones

925 showed almost no EE differentiation (C). Similarly, the combination of $k / u^{R N A i}$

926 with $s c^{R N A i}$ resulted in clones lacking EE differentiation (D). E Quantification of

$927 \mathrm{GFP}^{+} / \mathrm{Pros}^{+}$double-positive cells/clone of the genotypes in (A-D). $\mathbf{F}$.

928 Quantification of GFP ${ }^{+} /$Pros $^{+}$double-positive cells/clone of esg-F/O clones

929 expressing either UAS-sc, UAS-klu or the combination. See Figure S6 for

930 images. Error bars represent mean +/- S.D. Significance was calculated using

931 Student's t-test with Welch's correction. For E: $n=10$ for control and $s c^{R N A i}, n$

$932=14$ for $k l u^{R N A i}$, and $n=12$ for $s c^{R N A i} ; k l u^{R N A i}$. For F: $n=5$ for control, $n=6$ for

933 UAS-klu, UAS-sc and UAS-klu;UAS-sc.

934

935 Figure 6. Klu directly represses genes involved in Notch signaling, cell

936 cycle and EE differentiation. A-E. Klu-Dam binding tracks (in triplicate) for

937 the klu locus (A), sina locus (B), Cyclin $E(C)$ and Cyclin B (D) as well as the

$938 \mathrm{E}(\mathrm{Spl})$-complex genes $E(S p l)-m \delta$ and $E(S p l)-m \gamma(E)$. Tracks are displayed

939 using Integrated Genome Viewer (IGV) as overlayed tracks of the triplicate

940 Klu-Dam VS Dam-only control comparisons. Arrows indicate direction of

941 transcription. Numbers indicate the average maximum height of the peak

942 ( $\log _{2} \mathrm{FC}$ of Klu-Dam over Dam-only control) for each of the 3 replicates. $\mathbf{F}$.

943 Model of Klu function in lineage differentiation in the intestine. Klu expression

944 is activated by Delta-Notch signaling in the EB cell, together with other 
945 members of the Hairy/Enhancer of Split family of Notch target genes. Klu

946 accumulation in EBs results in a subsequent repression of these target genes,

947 including the repression of its own expression. Additionally, Klu acts as a

948 safeguard to repress erroneous EE differentiation in the enteroblast-

949 enterocyte lineage by indirectly repressing the accumulation of proneural

950 genes such as Asense and Scute through inhibition of the E3-complex

951 members Phyllopod ( $p h y l$ ) and Seven-in Absentia (sina) that repress the

952 accumulation of Scute and thereby inhibit EE fate. Finally, Klu acts in the

953 regulation of the cell cycle in EB cells, as the cell remodels its cell cycle from

954 a mitotic to an endocycle. 
bioRxiv preprint doi: https://doi.org/10.1101/590885; this version posted March 27, 2019. The copyright holder for this preprint (which was not

certified by peer review) is the author/funder, who has granted bioRxiv a license to display the preprint in perpetuity. It is made available under aCC-BY-ND 4.0 International license.

\section{Figure 1 Korzelius et al.}
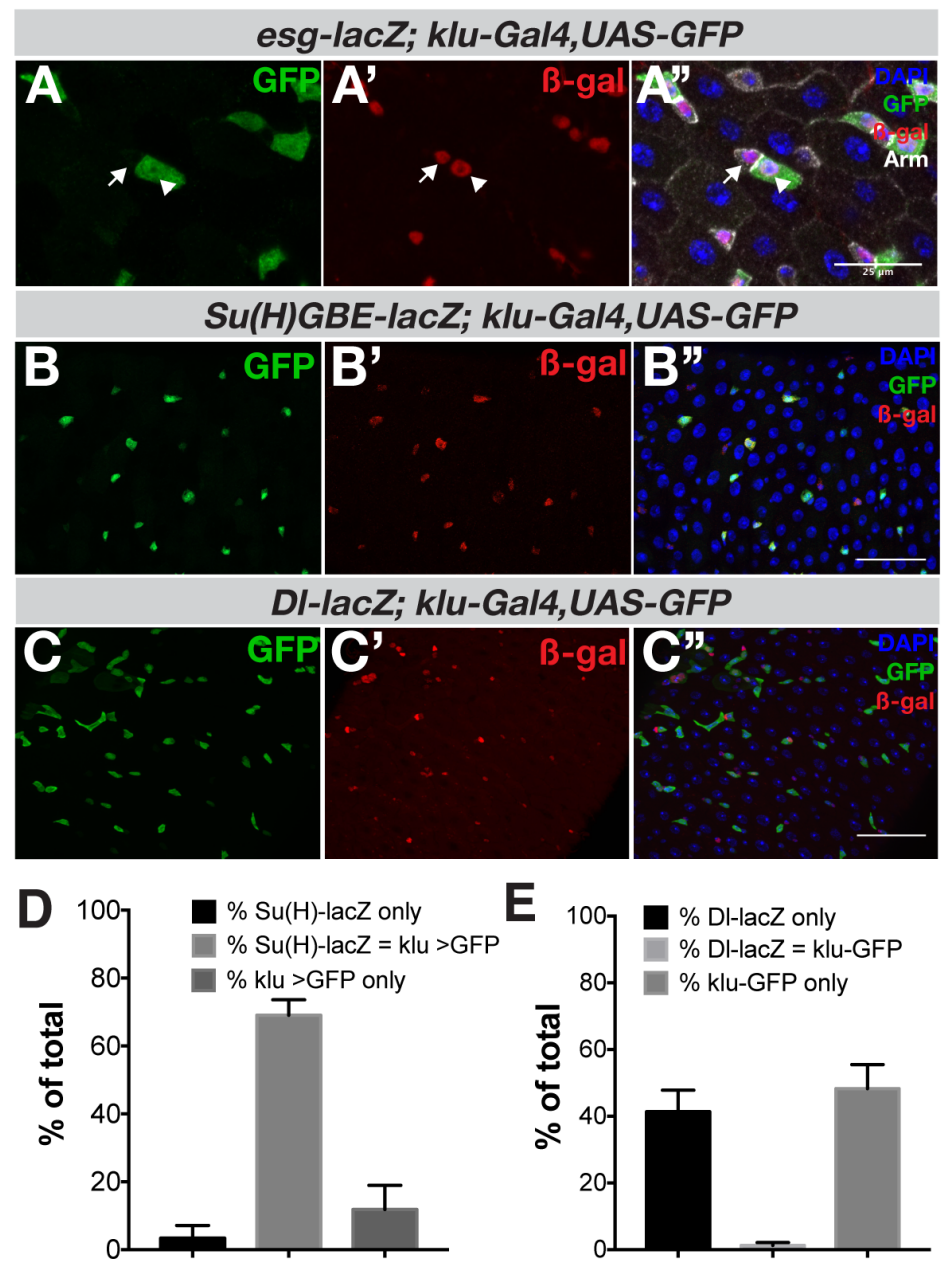

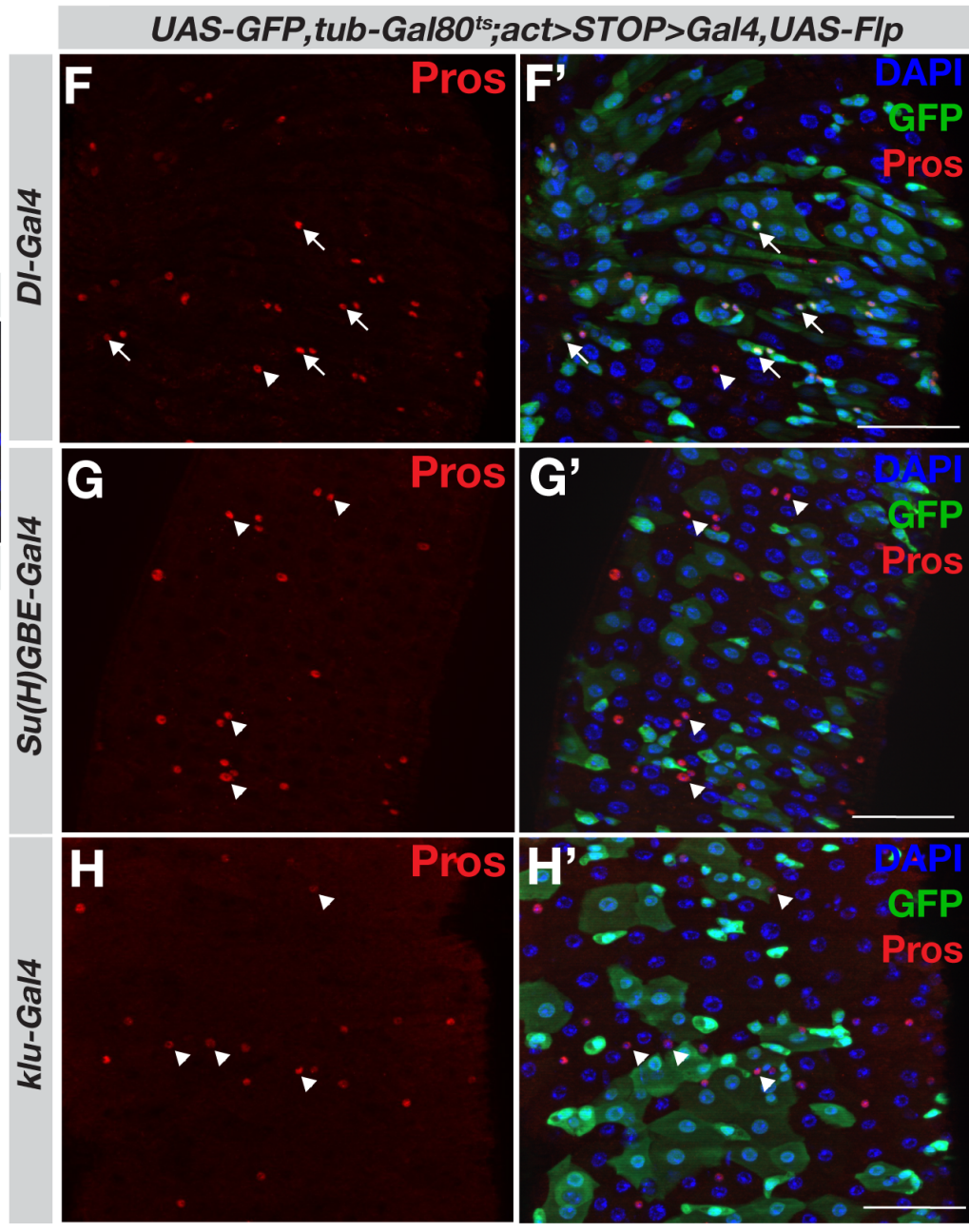


bioRxiv preprint doi: https://doi.org/10.1101/590885; this version posted March 27, 2019. The copyright holder for this preprint (which was not certified by peer review) is the author/funder, who has granted bioRxiv a license to display the preprint in perpetuity. It is made available under aCC-BY-ND 4.0 International license.

Figure 3 Korzelius et al.

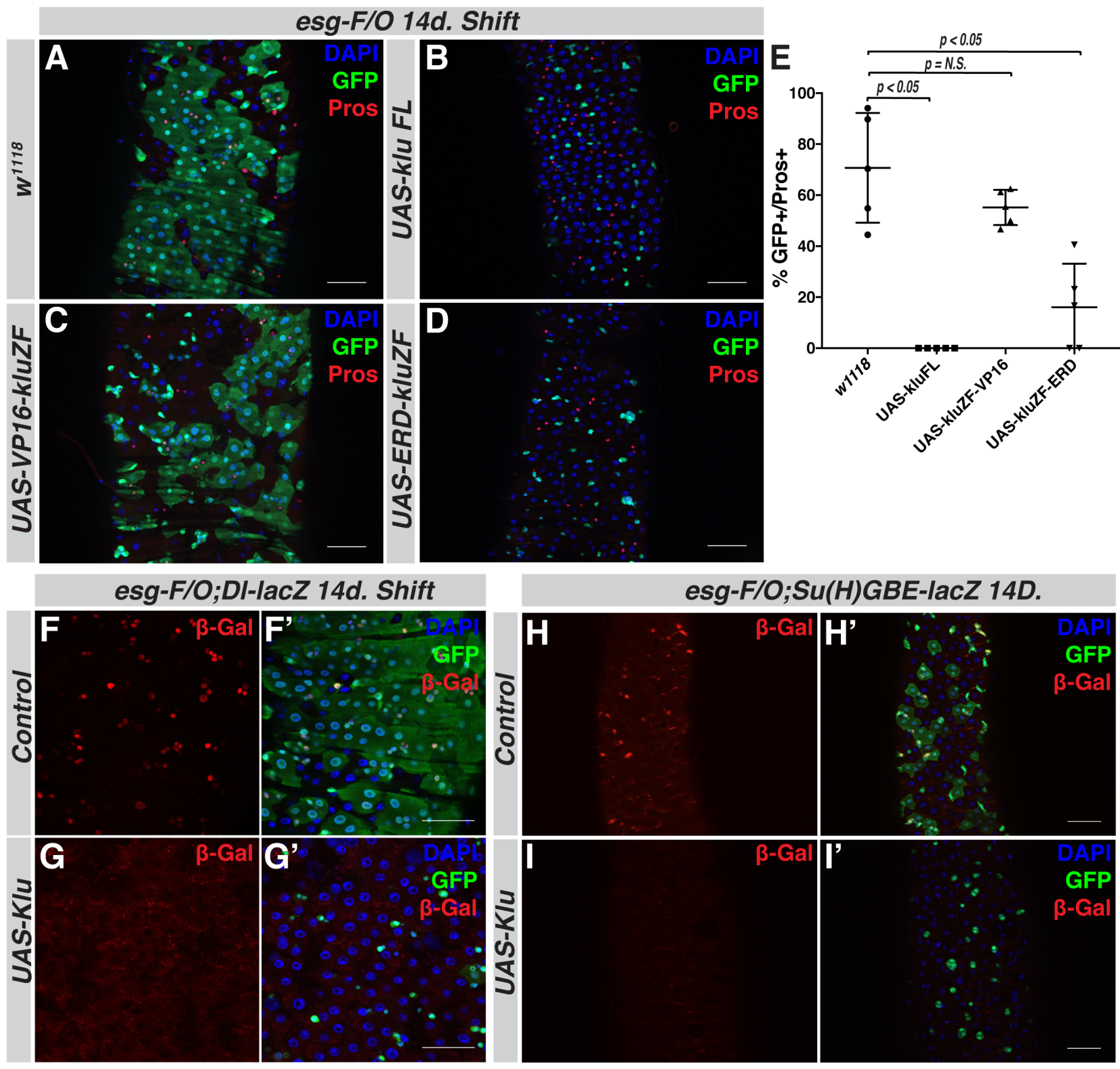




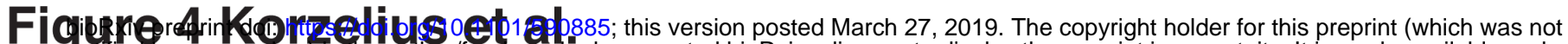
-ertitied by peer review) is the author/funder, who has granted bioRxiv a license to display the preprint in perpetuity. It is made available under

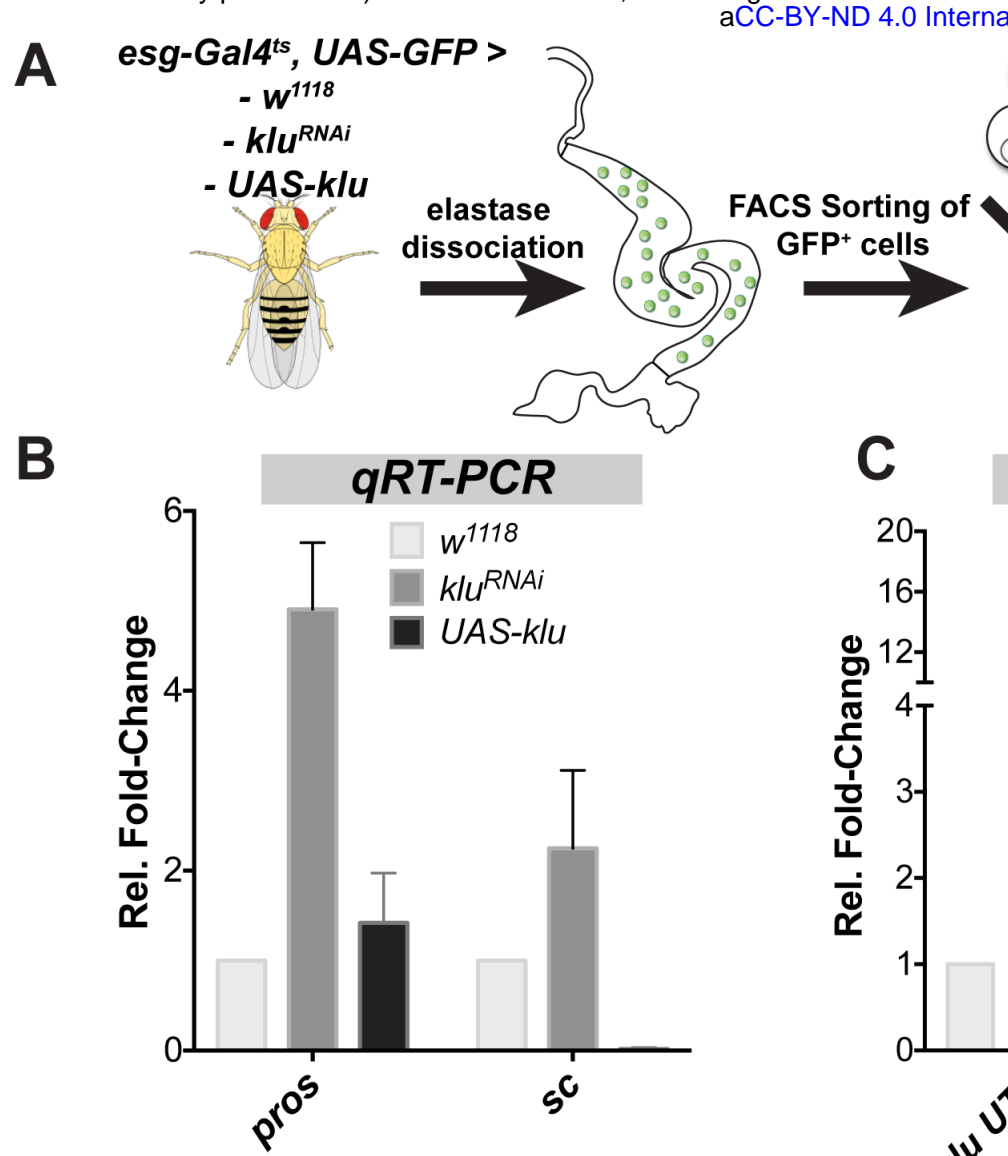
aCC-BY-ND 4.0 Internationak license.
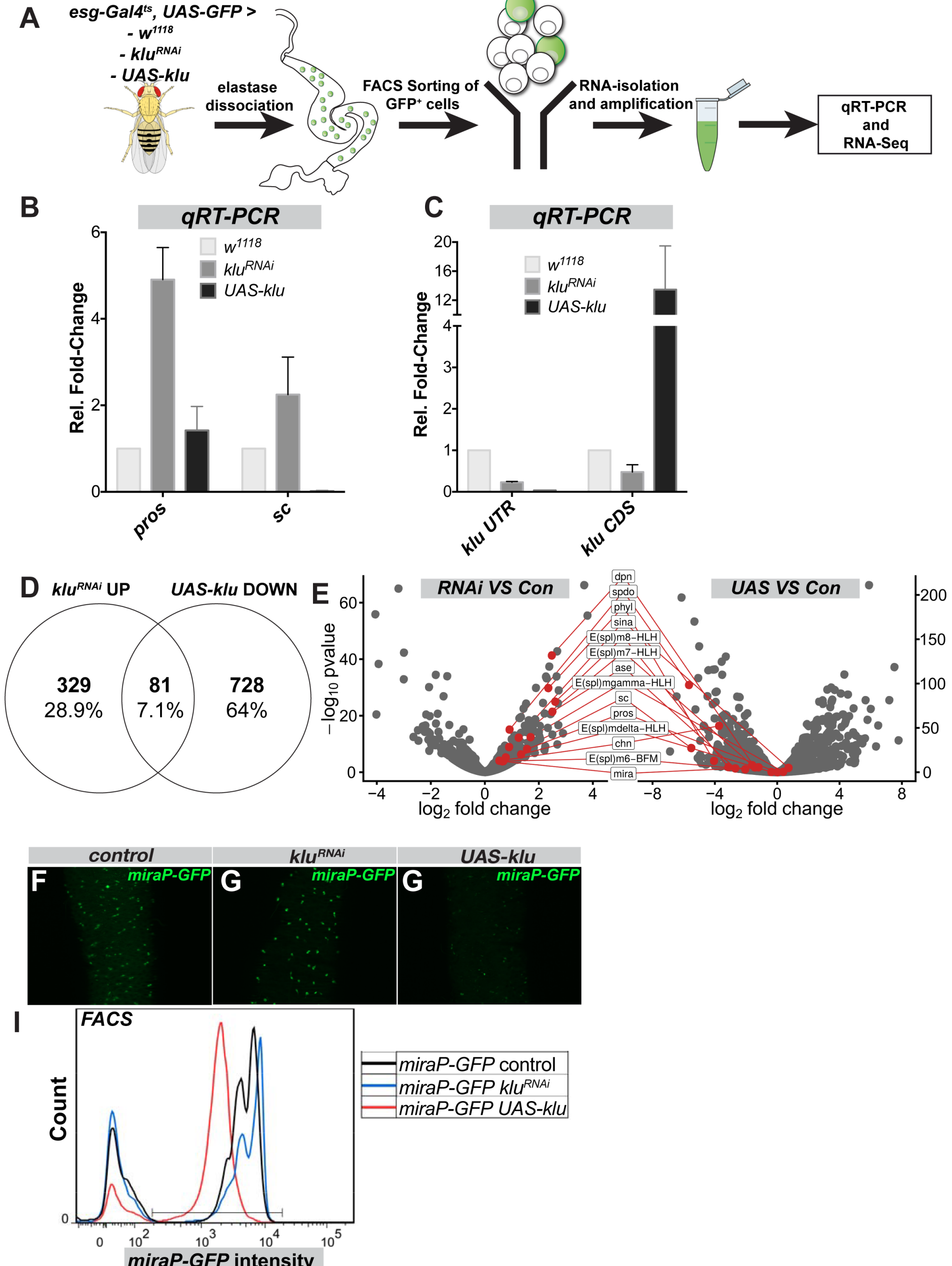

miraP-GFP control miraP-GFP klu RNAi miraP-GFP UAS-klu 
Figure 5 Korzelius et al.

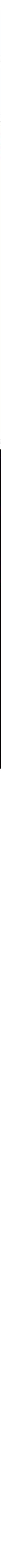


